\title{
Perfil e Qualidade de Vida de pacientes internados em hospital de custódia psiquiátrica
}

\author{
Plínio Pinto Malveira ${ }^{l}$; Glenda Arielle Guimarães Herrera ${ }^{2}$; \\ Raissa Araújo Rolim ${ }^{3}$; Ramiro Rolim Neto ${ }^{4}$; Gislene Farias Oliveira ${ }^{5}$
}

Resumo: A Reforma Psiquiátrica no Brasil trouxe uma série de mudanças na área da saúde mental, na perspectiva da regulamentação da desinstitucionalização, bem como dos instrumentos legais para a implementação de serviços alternativos em substituição aos existentes. Esta pesquisa objetivou conhecer e analisar o perfil e a qualidade de vida dos pacientes que estejam a cumprir alguma medida de segurança no Hospital Psiquiátrico Santa Tereza, localizado na cidade de Crato-CE. Trata-se de um estudo descritivo com sete custodiados, de um hospital psiquiátrico do interior do Ceará. Com relação à Qualidade de Vida, medida de acordo com os parâmetros da Organização Mundial de Saúde, percebeu-se que em todos os domínios, os respondentes da amostra apresentaram médias inferiores às médias descritas e padronizadas como normais.

Palavras-chave: Custódia psiquiátrica. Reforma psiquiátrica. Qualidade de vida.

\section{Profile and Quality of Life of patients admitted at the Custody Psychiatric Hospital}

\begin{abstract}
Psychiatric Reform in Brazil brought a number of changes in the mental health area, in view of the regulation of deinstitutionalization and legal instruments for the implementation of alternative service to replace existing ones. This research aimed to identify and analyze the profile and the quality of life of patients who are to accomplish any security measure in the Psychiatric Hospital Santa Tereza, located in Crato-CE. This is a descriptive study of seven under custody of a psychiatric Hospital in the state of Ceará. Regarding quality of life, measured according to the parameters of the World Health Organization, it was noticed that in all areas, sample respondents had lower averages those described averages and standardized as normal.
\end{abstract}

Keywords: Psychiatric custody. Psychiatric reform. Quality of life.

\footnotetext{
1 Médico pela Universidade Federal do Ceará - UFC. Email: plinio malveira@ hotmail.com;

2 Acadêmica de medicina pela Universidade Federal do Cariri - UFCA. E-mail: arielle_herrera@hotmail.com;

3 Médica pela Universidade Federal do Ceará - UFC. E-mail: Raissa_rolim@ @otmail.com;

4 Medico pela Universidade Federal de Campina Grande - UFCG.

5 Psicóloga. Doutora em Psicologia social pela UFPB. Pós doutorado em Ciências da saúde pela faculdade de Medicina do ABC - SP.

Email: gislenefarias@gmail.com.
} 
Id on Line Revista Multidisciplinar e de Psicologia

Id on Line Multidisciplinary Journal and Psycology

\section{Introdução}

A Reforma Psiquiátrica no Brasil trouxe uma série de mudanças na área da saúde mental, na perspectiva da regulamentação da desinstitucionalização, bem como dos instrumentos legais para a implementação de serviços alternativos em substituição aos existentes.

Portanto o Ministério da Saúde, definiu uma série de leis para o atendimento às novas exigências sobre a assistência psiquiátrica a pessoas portadoras de transtornos mentais (BRASIL, 2001), o que incluiu também as condições físicas das instituições candidatas a fazerem tal atendimento, bem como as características da equipe multidisciplinar, dentre outros mecanismos para a garantia da qualidade do atendimento prestado.

A lei de 06 de abril de 2001 - Lei No 10.216/2001 (BRASIL, 2001), que dispõe sobre a proteção e os direitos das pessoas portadoras de transtornos mentais e redireciona o modelo assistencial em saúde mental, passou a definir as novas exigências sobre a assistência prestada aos pacientes psiquiátricos, o que incluiu também uma série de adequações destas a uma melhor qualidade de atendimento.

Embora se reconheça avanços significativos na nova legislação, com relação a medidas de segurança envolvendo pacientes psiquiátricos, estas não foram questões devidamente esclarecidas e aprofundadas na Reforma Psiquiátrica Brasileira. Os hospitais de custódia, em geral, não estão devidamente integrados à rede do SUS, salvo algumas exceções. Portanto ainda é um desafio o fortalecimento de uma rede de atenção extra-hospitalar na assistência ao infrator com problemas mentais.

Diferente de outras enfermidades, os transtornos mentais se revestem de uma particularização em cada enfermo, dada a história de vida e da doença de cada um, o que dificulta o seu prognóstico e uma temporalidade de melhora. Portanto, o provimento de cuidados assistenciais torna-se algo impositivo para o doente, o qual, em poucas vezes, colabora de forma consciente para a sua recuperação.

O Estado do Ceará, conta com apenas um Hospital de Custódia Psiquiátrica: o Instituto Psiquiátrico Governador Stênio Gomes (IPGSG), que existe há 43 anos, e é a única unidade prisional, funcionando como Manicômio Judiciário no Ceará. Normalmente recebe 
Id on Line Revista Multidisciplinar e de Psicologia

Id on Line Multidisciplinary Journal and Psycology

custodiados que padecem com algum tipo de transtorno psíquico. Segundo reportagem do Diário do Nordeste, veiculada em 12 de dezembro de 2013, há atualmente 120 detentos nas dependências do Instituto, em Itaitinga, Região Metropolitana de Fortaleza. Muitos dos internos são abandonados pela família após os delitos. São detentos provenientes de diversos municípios do Estado, que vêm para obter tratamento, e não para cumprir pena. Comprovada a autoria dos crimes, há a necessidade dos devidos cuidados psiquiátricos, por isso a Justiça os envia para o IPGSG, até que cessem os sintomas dos transtornos, ou que cumpram a medida de segurança imputada (DIÁRIO DO NORDESTE, 2013).

O processo de organização dos serviços de saúde mental, em geral, são caracterizados por uma construção lenta e complexa. A falta de uma rede de assistência básica eficiente tende a gerar situações críticas, e, quando há aumento da demanda, os hospitais psiquiátricos ficam superlotados. Esta situação não é diferente no Hospital Santa Tereza em Crato, Ceará, instituição onde se desenvolverá a presente pesquisa.

Esta instituição tem-se prestado à função de hospital de custódia psiquiátrica na região do cariri Cearense, tanto pela distância do único hospital de custódia psiquiátrica do Estado como por ser o único hospital psiquiátrico na região. É uma instituição tanto de defesa social como de clínica psiquiátrica na região que atende. Tem-se prestado, em alguns casos, ao atendimento de pessoas portadoras de distúrbios mentais que cometeram algum ato delitivo e, por isso, foram submetidas judicialmente à custódia. Em tais casos, seu objetivo é oferecer um tratamento psiquiátrico ao paciente, garantindo a preservação dos seus direitos humanos, sua dignidade como pessoa e sua qualidade de vida, bem como o bom atendimento durante a internação. A ideia é a recuperação dos pacientes sob seus cuidados, numa tentativa de reintegrá-los posteriormente ao meio social e de custodiar os indivíduos que, por determinação judicial, receberam alguma medida de segurança a cumprir.

No entanto, muito pouco se sabe acerca de como estão vivendo esses indivíduos na instituição objeto do presente estudo, de como é o tratamento a que estão sujeitos e quais as perspectivas acerca de seu futuro. Dessa forma, julgamos justificada essa pesquisa. Acreditamos ser importante conhecer a realidade dessas pessoas, a fim de propor políticas públicas mais adequadas a um modo de viver mais humano, para essa clientela. 
Esta pesquisa objetivou conhecer e analisar o perfil e a qualidade de vida dos pacientes que estejam a cumprir alguma medida de segurança no Hospital Psiquiátrico Santa Tereza, localizado na cidade de Crato-CE. Foi necessário adicionalmente, relacionar a qualidade de vida com as variáveis sexo, idade, estado civil, doença atual, percurso penal, dentre outras, entre os sujeitos da amostra, bem como comparar se houve diferença entre os níveis de qualidade de vida entre os sujeitos.

A medida de segurança é a ação penal que é aplicável às pessoas com deficiência, doenças ou transtornos mentais que cometem crimes ou infrações e são consideradas inimputáveis ou semi-inimputáveis (BRASIL, Lei n 7.209, artigo 26, de 11 de julho de 1984; BRASIL, 1940; 1998). A execução da sanção penal consiste no isolamento terapêutico que será estabelecido por tempo indeterminado ou até quando for verificada a cessação de periculosidade por uma perícia médica, porém é exigido um prazo mínimo de um a três anos (BRASIL, Lei n ${ }^{\circ}$ 7.209, artigo 97, de 11 de julho de 1984).

Para a aplicação das medidas de segurança, é necessária uma perícia psiquiátrica, a qual atesta aos juízes a anormalidade mental do sujeito, no momento do crime ou infração, que o impediu de compreender a antijuridicidade do seu ato. Se a perícia conseguir confirmar a insanidade mental no momento do delito, e o juiz em questão acatar o resultado pericial, medida de segurança ambulatorial ou de internação será aplicada a depender do caso. (BRASIL, Lei nº 7.209, artigo 97, de 11 de julho de 1984).

A custódia psiquiátrica ou internação compulsória implica vigilância e cuidado (SILES et al, 2001) e é definida, em conformidade com a lei, por um juiz que considerará as condições de segurança do local, quanto à proteção do paciente, dos demais internados e dos funcionários (BRASIL, Lei n ${ }^{0}$ 10.216, artigo 9, de 6 de abril de 2001).

O condenado a quem sobrevém doença mental deve ser recolhido, devido a sua periculosidade, a Hospital de Custódia e Tratamento Psiquiátrico (HCTP) ou, na falta desse, a outro estabelecimento adequado, como Alas de Tratamento Psiquiátrico (ATP) localizadas em presídios comuns (BRASIL, Lei nº 7.209, artigo 41, de 11 de julho de 1984). 
Id on Line Revista Multidisciplinar e de Psicologia

Id on Line Multidisciplinary Journal and Psycology

\section{Material e Método}

Trata-se de um estudo descritivo, com uma amostra de pacientes que cumpriam pena, em regime fechado, na Casa de Saúde Santa Tereza em Crato-CE, em outubro de 2015. Tratase de um hospital psiquiátrico, único na região do cariri Cearense que se presta a esse tipo de serviço.

Foi fundado em 21 de junho de 1970 e, desse tempo até hoje, possui um acervo de sete mil prontuários psiquiátricos. Em muitos casos, de pacientes custodiados. Também funciona como hospital-escola, atendendo a estudantes de medicina e de enfermagem de faculdades e universidades da região.

A amostra será o universo das pessoas que preencherem os critérios de inclusão, a saber: estar internado, sob custódia, em regime fechado, ter aval do médico psiquiatra que o acompanha, isto é, apresentar condições de sanidade para compreender os propósitos do estudo e aceitar participar da pesquisa.

A coleta dos dados acontecerá no local de custódia dos sujeitos, onde os seus familiares, bem como a direção do hospital, serão informados dos objetivos do estudo, de forma a se obter um consentimento esclarecido das partes envolvidas.

Os instrumentos constam de uma escala de Qualidade de Vida WHOQOL-BREF (FLECK et al, 1999) e um questionário sócio demográfico.

\section{a) A Escala de Qualidade de Vida WHOQOL-bref}

Cientistas sociais, filósofos, políticos, profissionais de saúde, gestores educacionais, dentre outros seguimentos sociais, têm-se preocupado com conceitos como "padrão de vida" e "qualidade de vida", no sentido de valorizar parâmetros que dêem conta da diminuição da mortalidade, aumento da expectativa de vida e aumento de um maior aproveitamento da vida com potencial de vitalidade (FLECK et al, 1999).

As medidas de qualidade de vida podem fornecer informações sobre aspectos pessoais e sociais, bem como medidas de bem-estar psicológico, incorporando o ponto de vista das pessoas e focalizando e valorizando a avaliação do respondente mais do que qualquer outra coisa (HIGGINSON et al, 2001). 
A qualidade de vida é definida pela Organização Mundial da Saúde (OMS, 1997) como: "[...] a percepção do indivíduo de sua posição na vida no contexto da cultura e sistema de valores nos quais ele vive e em relação aos seus objetivos, expectativas, padrões e preocupações". Esta definição deixa implícita a idéia do conceito subjetivo, multidimensional e inclui elementos de avaliação tanto positivos como negativos (OMS, 1995). Também reflete a subjetividade do construto inserida no contexto cultural, social e do meio ambiente (FLECK et al, 2000).

A avaliação da qualidade de vida é um tema complexo, e o desenvolvimento de instrumentos de avaliação psicometricamente válidos, considerando-se ainda que a maioria deles foi desenvolvida nos Estados Unidos e na Europa, é um grande desafio (FLECK et al, 2000). Além disso, a percepção da qualidade de vida varia entre indivíduos e é dinâmica para cada pessoa.

Apesar das dificuldades, as avaliações podem proporcionar uma melhor compreensão sobre as reais necessidades das pessoas, tanto na sociedade em geral como na área da educação.

Um dos assuntos fundamentais em avaliação de qualidade de vida é determinar o que é importante para o indivíduo, especialmente quando o instrumento é para uso em diferentes culturas. Sobre isso, uma análise realizada pelo Grupo de Qualidade de Vida da OMS demonstrou que é possível desenvolver uma medida de qualidade de vida aplicável e válida para uso em diversas culturas e organizou um projeto colaborativo em 15 centros, cujo resultado foi a elaboração do World Health Organization Quality of Life-100 - WHOQOL100 (OMS, 1998).

Devido à necessidade de instrumentos curtos e de rápida aplicação, foi, então, desenvolvida a versão abreviada do WHOQOL-100, o WHOQOL-bref, cuja versão final ficou composta por 26 questões (FLECK et al). A primeira questão refere-se à qualidade de vida de modo geral e a segunda, à satisfação com a própria saúde. As outras 24 estão divididas nos domínios físico, psicológico, das relações sociais e meio ambiente, sendo um instrumento que pode ser utilizado tanto para populações saudáveis como para populações acometidas por agravos e doenças crônicas (GONÇALVES e VILARTA, 2004). Além do caráter 
transcultural, os instrumentos WHOQOL valorizam a percepção individual da pessoa, podendo avaliar qualidade de vida em diversos grupos e situações.

A versão em português foi realizada segundo metodologia preconizada pelo Centro WHOQOL para o Brasil e apresentou características psicométricas satisfatórias (FLECK et al). Esta versão é composta por vinte e seis itens que avaliam a Qualidade de Vida (por exemplo, como você avalia sua qualidade de vida; o quanto você aproveita a vida; em que medida você acha que sua vida tem sentido). Os participantes dão suas respostas em uma escala de 5 pontos, com os extremos 1 (muito ruim) e 5 (muito boa). (Anexo II).

\section{b) Informações sócio-demográficas}

Foi proposto um conjunto de perguntas visando caracterizar a amostra, a exemplo de idade, sexo, classe social estimada, escolaridade, profissão atual, dentre outras que caracterizem a amostra.

Antes da aplicação definitiva do questionário, foi realizada uma aplicação piloto, como forma de testar se o instrumento está adequado e se contém todas as questões compreensíveis, não dúbias.

Quanto ao procedimento, para o contato inicial com a instituição, de forma a se conseguir a permissão para realização do estudo, apresentar-se-á um ofício. Em seguida, farse-á um contato com os sujeitos e a família, explicando o propósito do estudo, com a finalidade de se obter o consentimento informado, de acordo com a resolução 466/12, que trata sobre experimentação com seres humanos.

A análise estatística foi feita através do programa SPSS 15.0.

\section{Considerações éticas}

O projeto atendeu as determinações do Comitê de Ética, através da Plataforma Brasil, para autorização do trabalho de campo.

Os sujeitos e seus familiares, bem como a direção do estabelecimento hospitalar serão devidamente esclarecidos do propósito do estudo, de acordo com a Resolução 466/12 do 
Conselho Nacional de Saúde (Brasil), que trata das diretrizes e de normas regulamentadoras de pesquisas envolvendo seres humanos.

Os dados irão permanecer sob a guarda do pesquisador, sendo garantidos o seu sigilo e a confiabilidade das informações.

\section{Resultados e Discussão}

\section{Perfil geral dos respondentes}

Foi objetivo do presente estudo, determinar o perfil e o nível de Qualidade de Vida dos pacientes que estavam a cumprir alguma medida de segurança no Hospital Psiquiátrico Santa Tereza em Crato, Ceará - Brasil. Para isso foi necessário também, relacionar a qualidade de vida dos sujeitos com variáveis como sexo, idade, estado civil, doença atual, percurso penal, dentre outras.

Fizeram parte deste estudo 7 custodiados psiquiátricos, do Hospital Santa Tereza de Crato, Ceará - Brasil, que não se opuseram à participação. A distribuição dos sujeitos de acordo com o sexo e a idade dos participantes, encontra-se na tabela 1.

Tabela 1: Distribuição do sexo e idade dos sujeitos da pesquisa

\begin{tabular}{lcccc}
\hline Profissão & \multicolumn{2}{c}{ Frequiência } & Percental (\%) \\
\hline Masculino & \multicolumn{2}{c}{05} & 71,4 \\
\hline Feminino & & 02 & 28,6 \\
\hline Total & Mínimo & Máximo & Média & Desvio padrão \\
\hline & 19 & 74 & 41,14 & 18,986 \\
\hline Idade & 19 & & $\mathbf{1 0 0 , 0}$ \\
\hline
\end{tabular}

Crato/CE, 2015 
As idades variaram entre 19 e 74 anos (média 41,14; Dp=18,986), sendo apenas um sujeito com idade até 19 anos $(14,3 \%)$. A maioria do sexo masculino $(71,4 \%)$, num total de 7 custodiados psiquiátricos.

Para uma melhor visualização, a distribuição do sexo dos sujeitos, encontra-se no gráficos 1 a seguir:

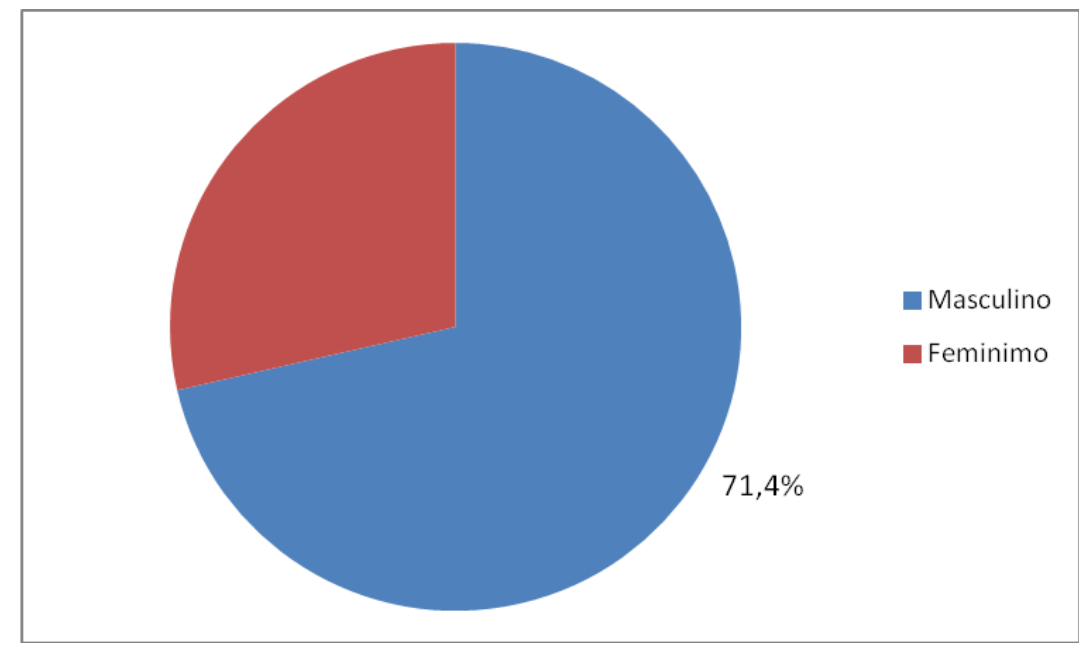

Gráfico 1 - Sexo dos participantes

O Gráfico 1 mostra que os respondentes foram em sua maioria do sexo masculino. Quanto a idade, a distribuição foi relativamente normal (19, 27, 31, 39, 40, 58 e 74 anos de idade).

A idade é um fator importante na observação da Qualidade de Vida. Estudos recentes de Costa et al., (2003) demonstraram que, entre pessoas do sexo feminino, na faixa de 30 a 39 anos, os níveis de Qualidade de vida são os mais baixos.

Acreditou-se necessário conhecer também sobre a Escolaridade dos respondentes. Os resultados estão dispostos na tabela 2 a seguir: 
Id on Line Revista Multidisciplinar e de Psicoloqia

Id on Line Multidisciplinary Journal and Psycology

Tabela 2: Escolaridade dos sujeitos da pesquisa

\begin{tabular}{lcc}
\hline Escolaridade & Freqüência & Percentual (\%) \\
\hline Sem Instrução formal & 1 & 14,0 \\
\hline Ensino Fundamental Incompleto & 6 & 86,0 \\
\hline Total & $\mathbf{7}$ & $\mathbf{1 0 0 , 0}$ \\
\hline
\end{tabular}

Crato-CE, 2015

Para uma melhor visualização, a formação dos participantes foi disposta no gráfico 2, a seguir.

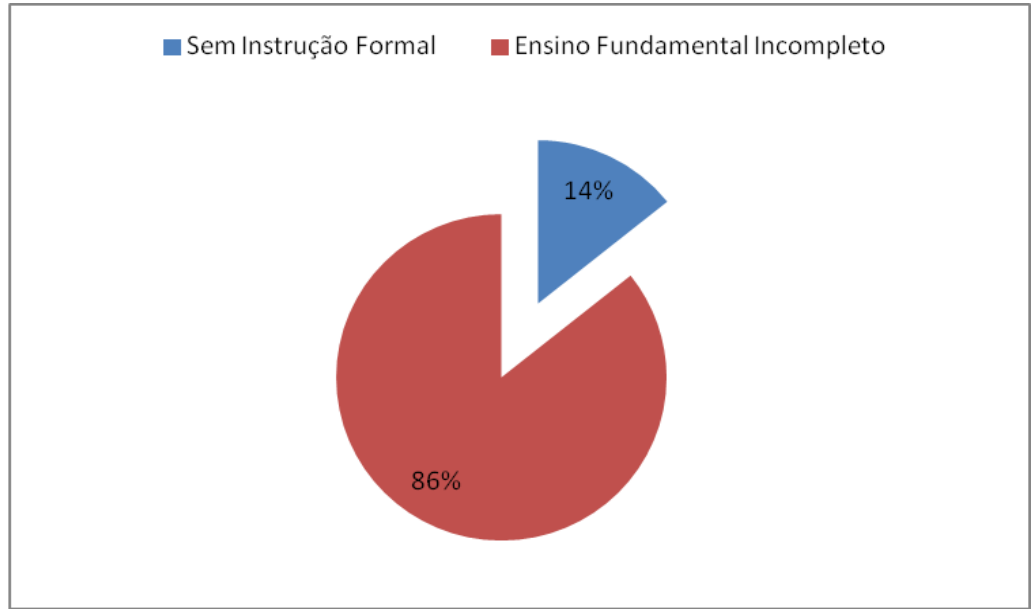

Gráfico 2 - Distribuição da Escolaridade dos participantes

Quanto à escolaridade, a amostra se apresentou da seguinte forma: um sujeito sem instrução formal $(14,3 \%)$ e seis sujeitos com o Ensino Fundamental incompleto $(85,7 \%)$. Portanto pessoas com nível de escolaridade muito precária.

Outro aspecto julgado importante foi a profissão dos sujeitos, dispostas na tabela 3 a seguir. 
Id on Line Revista Multidisciplinar e de Psicoloqia

Id on Line Multidisciplinary Journal and Psycology

Tabela 3: Profissão dos sujeitos da pesquisa

\begin{tabular}{lcc}
\hline Profissão & Freqüência & Percentual (\%) \\
\hline Doméstica & 1 & 14,3 \\
\hline Jardineiro & 1 & 14,3 \\
\hline Agricultor & 4 & 57,1 \\
\hline Não trabalha & 1 & 14,3 \\
\hline Total & $\mathbf{7}$ & $\mathbf{1 0 0 , 0}$ \\
\hline
\end{tabular}

Crato-CE, 2015

Percebe-se que os entrevistados, além de estarem numa situação de confinamento, exerciam funções de baixa remuneração associada à baixa escolaridade. Acredita-se serem estes determinantes importantes para uma diminuição na qualidade de vida dessas pessoas.

Quando indagados sobre quem era o responsável por eles, 71,4\% responderam que eram seus pais, $14,3 \%$ responderam que era outro parente e $14,3 \%$ responderam que era outra pessoa. Quanto ao estado civil, dos 7 entrevistados, 58,1\% eram solteiros, $27,6 \%$ eram viúvos e $14,3 \%$ eram divorciados. Portanto, a totalidade dos entrevistados encontrava-se sem um relacionamento estável, o que, de acordo com Mascarenhas, Prado e Fernandes (2013), implicaria em menor qualidade de vida para os pacientes.

O tempo de custódia variou de três meses a um ano e oito meses de reclusão, sendo a média de oito meses. Nenhum dos detentos sabia por quanto tempo ainda permaneceriam sob custódia. Quando indagados se esta era a primeira vez que cumpriam pena em regime de custódia psiquiátrica, $85,7 \%$ dos entrevistados responderam que estavam cumprindo pena pela primeira vez. Segue a tabela com os resultados:

Tabela 4: Primeira vez em Custódia Psiquiátrica?

\begin{tabular}{lcc}
\hline & Freqüência & Percentual (\%) \\
\hline SIM & 6 & 85,7 \\
\hline NÃO & 1 & 14,3 \\
\hline Total & $\mathbf{7}$ & $\mathbf{1 0 0 , 0}$ \\
\hline
\end{tabular}

Crato - CE, 2015 
Quanto aos tipos de delitos praticados, estes consistiram em ameaça, agressão, tentativa de estupro, agressão com troca de tiros, uso de drogas, extorsão, promoção de incêndio e estupro. Indagados se já haviam sido internados outras vezes por alguma enfermidade mental, $4(57,1 \%)$ responderam que sim e $3(42,9 \%)$ responderam que não. Portanto, uma maioria de indivíduos com um histórico anterior de problemas psiquiátricos.

Com relação a auto-avaliação da Qualidade de vida, medida através da questão 1 do WOHQOL, 28,6\% avaliaram como ruim, 14,3\% como ruim e 57,1\% como boa. Quanto ao nível de satisfação com a sua saúde (questão 2 do WOHQOL), de maneira geral, 28,6\% se autodeclararam muito insatisfeitos, $14,3 \%$ se autodeclararam insatisfeitos, $14,3 \%$ se autodeclararam nem insatisfeito e nem satisfeito e $42,9 \%$ se declararam satisfeitos.

A saúde e o suporte social têm sido considerados em diversos estudos bons prognósticos da qualidade de vida (VISWERVARAN, SANCHEX E FISHER, 1999). No caso do presente estudo, percebe-se que, pelo menos, metade dos sujeitos afirmou estar insatisfeito ou muito insatisfeito com sua saúde atual.

Com relação aos domínios do WHOQOL abreviado, as respostas estão consolidadas na tabela a seguir:

Tabela 5: Dimensões da Qualidade de vida dos participantes do estudo.

\begin{tabular}{|l|c|c|c|c|c|}
\hline & $\begin{array}{c}\text { Domínio } \\
\text { Físico }\end{array}$ & $\begin{array}{c}\text { Domínio } \\
\text { Psicológico }\end{array}$ & $\begin{array}{c}\text { Domínio } \\
\text { Social e de } \\
\text { Relações }\end{array}$ & $\begin{array}{c}\text { Domínio } \\
\text { Ambiental }\end{array}$ & $\begin{array}{c}\text { Qualidade } \\
\text { de Vida } \\
\text { Global }\end{array}$ \\
\hline Média & 19,57 & 20,28 & 11,28 & 29,42 & 80,57 \\
\hline Desvio Padrão & 3,25 & 4,38 & 2,36 & 5,41 & 10,78 \\
\hline Mínimo & 14,00 & 14,00 & 8,00 & 22,00 & 67,00 \\
\hline Máximo & 23,00 & 27,00 & 15,00 & 37,00 & 99,00 \\
\hline
\end{tabular}

Crato - CE, 2015

Com relação aos Domínios de Qualidade de Vida, os respondentes se perceberam como pessoas mais saudáveis no Domínio Ambiental (média 29,42) e menos saudáveis no Domínio Social e de Relações (média 11,28), no Domínio Físico (média 19,57) e no Domínio 
Psicológico (média 20,28) do WHOQOL. Em termos de Qualidade de Vida Global, a média foi de 80,57 com $\mathrm{DP}=10,78$.

Com relação à Qualidade de Vida, medida de acordo com os parâmetros da Organização Mundial de Saúde, percebeu-se que em todos os domínios, os respondentes da amostra apresentaram médias inferiores às médias descritas e padronizadas como normais.

Apesar da Qualidade de Vida Global demonstrar uma pontuação média superior a proposta pela OMS, que é de 64 pontos, as evidências nos Domínios Parciais indicaram o contrário. Tais resultados nos levam a crer que o confinamento, aliado a um comprometimento real da saúde no âmbito físico e no de relacionamentos, parece ser gerador de problemas reais como desinteresse, falta de significado de vida, falta de motivação pela insegurança gerada em não saber por quanto tempo permanecerá sob custódia, implicando em uma queda na QV.

Concluímos que, quanto ao significado de estar confinado sob custódia psiquiátrica, embora atenda a necessidades básicas de alimentação e segurança, estas parecem não serem suficientes quando se estuda as dimensões separadamente. Porém, de uma forma Global, os respondentes parecem apresentar uma QV superior ao preconizado pela OMS. Este fato poderia ser mais bem explicado por conta de alguma medicação neuroléptica, comuns em Hospitais Psiquiátricos, as quais podem ter provocado respostas mais positivas, em termos de bem-estar subjetivo.

No que se refere às Representações Sociais de Doença mental, sobressaíram-se duas Categorias: uma Negativa e outra Positiva, embora as respostas estiveram, na sua quase totalidade, associadas a algo Negativo, sobressaindo-se duas sub-Categorias: a) Física 'tratamento', 'doença', 'adrenalina', 'sistema nervoso afetado' (2), 'loucura', 'ferida', 'pessoa louca', 'fragilidade de cabeça', 'coisa alterada de pressão' (2), 'zumbidos', 'ansiedade' e b) Social - 'não consegue se sustentar sozinho', 'pessoa fica mais ou menos', ‘quebrando', 'batendo nos outros', 'espancando', ‘quer ser liberta da agoniação', 'tumulto', 'roubar', 'matar', 'correr na pista', 'coisas que não deve'. Positiva - 'normal', 'não é mal', 'quer ser liberta'. 


\section{Conclusão}

Diante dos dados apresentados, torna-se clara a necessidade de investimentos em estratégias para assistência, promoção e pesquisa sobre QV, tanto do âmbito jurídico como dos serviços de saúde mental, em vista das necessidades dos portadores de transtorno mental em ação penal. Segundo a OMS, "a nossa saúde mental tem um impacto opressivo em nossas habilidades para funcionar e participar na sociedade. Portanto, temos de começar a colocar mais de nossos recursos a favor da saúde mental”. Nessa perspectiva, políticas públicas que contemplem essas áreas necessitam ser incentivadas e colocadas em prática nas instituições de saúde mental, como a Casa de Saúde Santa Tereza.

Para se alcançar mudanças positivas, as decisões nas instituições têm de ser baseadas em evidências científicas sobre a abordagem e o tratamento que mantenham a saúde mental, para que, só assim, haja alteração das políticas de benefícios e dos recursos humanos direcionados (MORENO-JIMENEZ, 2000). Dessa forma, estudos comparativos necessitam ser conduzidos, de forma a auxiliar na construção de indicadores que subsidiem a escolha da melhor estratégia terapêutica para essa população, ou seja, se tratamento ambulatorial ou em regime fechado. Há também a necessidade de que instrumentos específicos que contemplem a avaliação do tratamento e da QV de pacientes em cumprimento de MS sejam desenvolvidos.

A interlocução entre o sistema prisional e o de saúde pública no Brasil tem sido apontada pela literatura como um importante meio para a evolução da saúde coletiva (RICH JD, 2007). O serviço prestado nas unidades de tratamento em saúde mental ligadas ao poder judiciário precisa ser avaliado e comparado ao serviço prestado nas unidades civis de atenção, a fim de que possa ser promovida uma maior interação entre os modelos de tratamento, bem como para que os princípios e as propostas da Reforma Psiquiátrica possam alcançar e beneficiar os pacientes em medida de segurança.

Novos estudos precisam ser realizados nas instituições brasileiras para que seja possível uma mudança de paradigma em relação à periculosidade e à inimputabilidade dos portadores de transtorno psiquiátrico. Estudos dessa natureza podem contribuir para a modificação do estigma da periculosidade e para a viabilização de políticas compatíveis com um tratamento mais digno e humanitário para tais pacientes. 
Por fim, o desenvolvimento do conhecimento empírico do constructo qualidade de vida é tarefa ainda a ser realizada pela ciência. Sem essa tarefa, a ênfase sobre a Q.V. das pessoas pode tornar-se simples retórica. $\mathrm{O}$ interesse por qualidade de vida indica, talvez, o reconhecimento do grau que a sociedade se interessa por garantir a todos os seus membros o acesso aos seus direitos e responsabilidades e, dessa forma, refletir sua humanidade. Dessa maneira, apenas avanços conceituais e metodológicos possibilitarão aplicações das medidas de Q.V. de forma que responda à sociedade, à política de saúde, ao grupo de pacientes e ao paciente individual, e, assim, proporcionar avanços significativos na melhoria de vida das populações e, de forma mais direcionada, dos pacientes em custódia psiquiátrica, visto que os mesmos parecem necessitar de maior Satisfação e Qualidade de vida em relação ao que estão tendo durante a internação. Fatores que contribuem significativamente para dar sentido às nossas vidas.

\section{Referências}

BRASIL. Lei n. ${ }^{\circ} 10.216$ de 06 de abril de 2001. Dispõe sobre a proteção e os direitos das pessoas portadoras de transtornos mentais e redireciona o modelo assistencial em saúde mental. Disponível em:< http://www.planalto.gov.br/ccivil_03/leis/leis_2001/110216.htm.>. Acesso em: 07 dez. 2013

BRASIL. Código Penal. Decreto-lei no 2.848 de 07.12.1940 alterado pela Lei $\mathrm{n}^{\circ} 9.777$ em 26/12/98

BRASIL. Lei n. ${ }^{\circ}$ 7.209 de 11 de julho de 1984. Altera dispositivos do Decreto-Lei $\mathrm{n}^{\circ} 2.848$, de 7 de dezembro de 1940 - Código Penal, e dá outras providências. Disponível em: $<$ http://www.planalto.gov.br/ccivil_03/Leis/1980-1988/L7209.htm>. Acesso em: 07 dez. 2013.

SILES et al. De la custodia a los cuidados: una perspectiva histórica de la enfermería en salud mental. Espanha, 2001.

BRASIL. Lei n. ${ }^{\circ} \mathbf{1 0 . 2 1 6}$ de 6 de abril de 2001. Dispõe sobre a proteção e os direitos das pessoas portadoras de transtornos mentais e redireciona o modelo assistencial em saúde mental. Disponível em:〈http://www.planalto.gov.br/ccivil_03/Leis/LEIS_2001/L10216.htm>. Acesso em: 07 dez. 2013. 
Id on Line Revista Multidisciplinar e de Psicologia

Id on Line Multidisciplinary Journal and Psycology

FLECK, Marcelo. O instrumento de avaliação de qualidade de vida da Organização Mundial da Saúde (WHOQOL-100): características e perspectivas. Ciência \& Saúde Coletiva, 5(1):33-38, 2000.

WORLD HEALTH ORGANIZATION. WHOQOL: Measuring quality of life. Genebra: WHO; 1997. MAS/MNH/PSF/97.4

WHOQOL Group. The World Health Organization Quality of Life Assessment (WHOQOL), 1995: position paper from the World Health Organization. Social Science and Medicine. V.41,n.10, P.403-409.

FLECK MPA et al. O instrumento de avaliação de qualidade de vida abreviado da Organização Mundial da Saúde (WHOQOL-breve): aplicação da versão em português. Revista de Saúde Pública. 2000; 22(2), (no prelo).

FLECK MP, LEAL OF, LOUZADA S, XAVIER M, CHACHAMOVICH E, VIEIRA G, et al. Desenvolvimento da versão em português do instrumento de avaliação de qualidade de vida da OMS (WHOQOL-100). Revista de Saúde Pública. 1999; 33(2):198-205.

HIGGINSON IJ, CARR AJ, et al. Using quality of life measures in the clinical setting. BMJ 2001;322:1297. http://dx.doi.org/10.1136/bmj.322.7297.1297

The WHOQOL Group 1998. The World Health Organization quality of life assessment (WHOQOL): development and general psychometric properties. Social Science and Medicine $12: 1569-1585$.

FLECK MPA et al. 2000. O instrumento de avaliação de qualidade de vida abreviado da Organização Mundial da Saúde (WHOQOL-breve): aplicação da versão em português. Revista de Saúde Pública 22(2), (no prelo)

VILARTA, Roberto; GONÇALVES, Aguinaldo Qualidade de Vida - concepções básicas voltadas à saúde. In: GONÇALVES, Aguinaldo e VILARTA, Roberto (orgs.). Qualidade de Vida e atividade física: explorando teorias e práticas. Barueri: Manole, 2004, p.27-62.

FLECK MPA et al. 2000. O instrumento de avaliação de qualidade de vida abreviado da Organização Mundial da Saúde (WHOQOL-breve): aplicação da versão em português.

Revista de Saúde Pública 22(2),(no prelo)

MASCARENHAS, Claudio Henrique Meira; PRADO, Fabio Ornellas; FERNANDES, Marcos Henrique. Fatores associados à qualidade de vida de Agentes Comunitários de Saúde. Ciênc. saúde coletiva, Rio de Janeiro, v. 18, n. 5, May 2013

VISWERVARAN, C., SANCHEX, J., \& FISHER, J. (1999). The role of social support in the process of work stress: A meta-analysis. Journal of Vocational Behaviour, 54, 314-334. 
MORENO-JIMENEZ, B. Olvido y recuperacón de los factores psicosociais em la salud laboral. Editorial dos Archivos de Prevención de Riesgos Laborales 3: 3-4, 2000

Rich JD. Health Issues in prisons and jails: implications for urban health. Journal of Urban Health. 2007;84(3):316-8.

\section{Como citar este artigo (Formato ABNT):}

MALVEIRA, P.P.; HERRERA, G.A.G.; ROLIM, R.A.; ROLIM NETO, R. OLIVEIRA, G.F. Perfil e Qualidade de Vida de pacientes internados em hospital de custódia psiquiatrica. Id on Line Revista Multidisciplinar e de Psicologia, Set-Out de 2016, vol.10, n.31, Supl 2, p. 177-193 . ISSN 1981-1179.

Recebido: 22/09/2016

Aceito: 29/09/2016 